\title{
Adjustment of the formula of local surface water quality assessment
}

\author{
Hung V. Bui*, \& Nhi M. U. Tran \\ Department of Environmental Management, University of Science, Ho Chi Minh City, Vietnam
}

ARTICLE INFO
Research Paper
Received: April 05, 2018
Revised: July 25, 2018
Accepted: August 13, 2018
Keywords
Surface water
Water quality
Water quality assessment
Water quality index
879/QD-TCMT
*Corresponding author
Bui Viet Hung
Email: bvhung@hcmus.edu.vn

\begin{abstract}
Water quality index (WQI) is widely applied for the assessment of water quality (WQ) according to decision No. 879/QD-TCMT of the Ministry of Natural Resource and Environment in most of the provinces. During the application process, the WQI has revealed many problems, such as inacculate assessment of water quality status, not addressing locally economic, social and environmental conditions, and easy misleading when using abnormally increaed values,... To resolve the above problems, the paper presents the steps to adjust the water quality index based on the current water quality components of the old WQI formula, the fuzzy comprehensive evaluation theory with the Entropy rate method. With the surface water quality data series and the status of the water use in the main river canal system in Vinh Long City from 2012 to 2017, the computation steps of coefficients and rates of the assessing water quality components are identified; through the new assessment water quality formula is adjusted and tested.
\end{abstract}

Cited as: Bui, H. V., \& Tran, N. M. U. (2018). Adjustment of the formula of local surface water quality assessment. The Journal of Agriculture and Development 17(5), 102-113. 


\title{
Điều chỉnh công thức đánh giá chất lượng nước mặt khu vực
}

\author{
Bùi Việt Hưng* \& Trần Minh Uyển Nhi
}

Bộ Môn Quản Lý Môi Trường, Trường Đại Học Khoa Học Tự Nhiên TP. Hồ CHí Minh, TP. Hồ Chí Minh

\section{THÔNG TIN BÀI BÁO}

Bài báo khoa học

Ngày nhận: 05/04/2018

Ngày chỉnh sửa: $25 / 07 / 2018$

Ngày chấp nhận: 13/08/2018

\section{Từ khóa}

Chất lượng nguồn nước

Chỉ số chất lượng nguồn nước

Đánh giá chất lượng nguồn nước

Nước mặt

879/QD-TCMT

*Tác giả liên hệ

Bùi Việt Hưng

Email: bvhung@hcmus.edu.vn

\section{TÓM TẮT}

Chỉ số chất lượng nước (WQI) được áp dụng rộng rãi cho đánh giá chất lượng nước (CLN) theo Quyết định số 879/QD-TCMT của bộ Tài nguyên Môi trường tại hầu hết các tỉnh thành. Trong quá trình áp dụng, chỉ số WQI đã bộc lộ nhiều bất cập như không phản ánh đúng thực trạng chất lượng nguồn nước, không phản ánh tính địa phương về kinh tế, xã hội và môi trường, và dễ bị sai lệch khi số liệu đưa vào tính toán có giá trị cao đột biến,... Để giải quyết các vấn đề trên, bài báo trình bày cách điều chỉnh công thức chỉ số CLN dựa trên các thành phần chất lượng của chỉ số WQI, cơ sở lý thuyết toán đánh giá toàn diện "mờ" với phương pháp xác định trọng số Entropy. Với bộ số liệu CLN và khảo sát thực trạng sử dụng nước mặt trên mạng sông kênh rạch chính khu vực Thành phố Vĩnh Long thu thập từ năm 2012 đến 2017, các bước tính toán các hệ số và tỷ trọng của các thành phần CLN mặt được xác định, qua đó điều chỉnh công thức chỉ số WQI.

\section{1. Đặt Vấn Đề}

Trong công tác quản lý chất lượng nguồn nước, đặc biệt là nguồn nước mặt, hầu hết các báo cáo chất lượng môi trường nước của các tỉnh thành trên cả nước đều sử dụng chỉ số WQI với cách thức tính toán theo hướng dẫn của Tổng cục Môi trường, bộ Tài nguyên và Môi trường trong Quyết định số 879/QĐ-TCMT (VEA, 2011).

Qua thực tiễn thực hiện nhiều đơn vị quản lý đã nhận thấy việc áp dụng chỉ số WQI cho tính toán gặp khá nhiều bất cập. Một trong số đó là chỉ số WQI phụ thuộc nhiều vào số thành phần chất lượng và mức độ chính xác của đo đạc chúng như hiện tượng cao biến, thiếu thông tin và nhiều thành phần không phản ánh đúng đặc điểm phát triển kinh tế, xã hội và môi trường của địa phương. Bên cạnh đó, do chỉ số WQI dựa vào số liệu đo đạc thô (đo đạc trực tiếp) quá lớn, chỉ số WQI thường bị thiên lệch hay "dẫn dắt" bởi các giá trị thành phần có tính chất đột biến (quá lớn hay mang tính chủ quan trong đo đạc).
Theo Che (2013), cách tính chỉ số WQI dựa trên phương pháp luận không hợp lý và không chắc chắn khi kết luận bậc chất lượng vì chỉ dùng một chỉ số định lượng cố định làm điểm phân chia.

Sơ đồ tính toán chỉ số chất lượng nguồn nước theo chỉ số WQI (truyền thống) theo quyết định số 879/ĐQ-TCMT được thể hiện theo Bảng 1.

Do vậy, cách đánh giá WQI không đưa ra được kết luận chung về chất lượng nguồn nước của khu vực trong thời đoạn thời gian như tháng, quý hay năm và nhiều năm. Cần có giải pháp cải tiến trong việc sử dụng số liệu thực đo một cách phù hợp, logic nhằm có thể khắc phục được những hạn chế của chỉ số WQI. Theo nhóm tác giả, việc áp dụng WQI trong đánh giá chất lượng nguồn nước mặt nên được tiếp tục với việc điều chỉnh công thức tính sao cho vẫn duy trì các ưu điểm trên và khắc phục được một số bất cập đã chỉ ra. Với quyết định 711/QĐ-TCMT của Tổng cục Môi trường điều chỉnh công thức WQI áp dụng cho khu vực sông Nhuệ Đáy và sông Cầu, đây thực sự là một hướng tiếp cận cho việc xác lập 
Bảng 1. Cách tính WQI truyền thống cho từng điểm đo và thời điểm đo

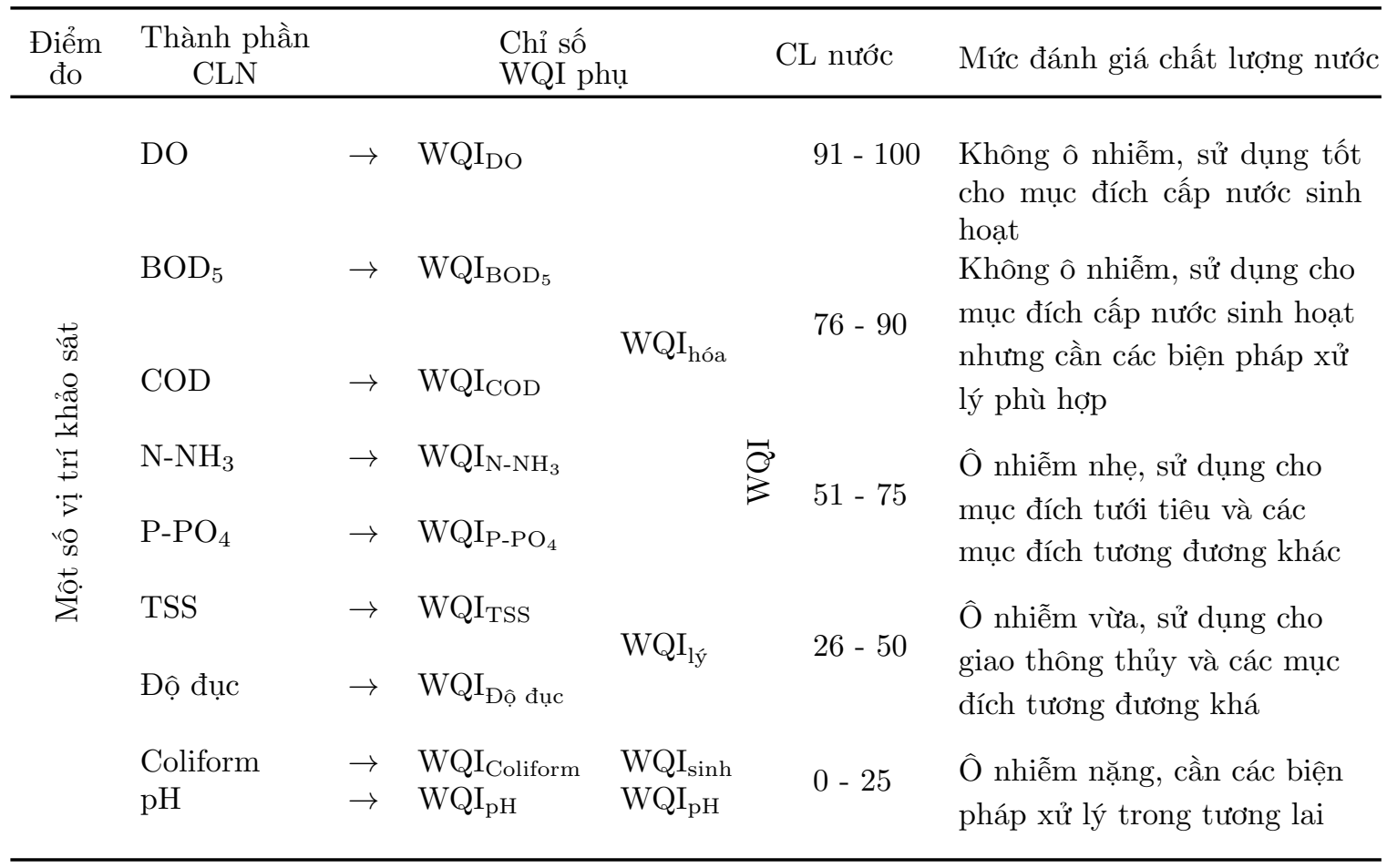

công thức tính chỉ số chất lượng nguồn nước mặt áp dụng cho từng địa phương, vùng với các đặc điểm đặc trưng.

Lý thuyết tập hợp mờ được sáng lập bởi nhà nghiên cứu toán học Zadeh, thuộc đại học California Berkeley - Mỹ (Alex, 2009). Ý tưởng nổi bật của khái niệm tập hợp mờ của Zadeh là từ những khái niệm trừu tượng về ngữ nghĩa của thông tin mờ, không chắc chắn như ô nhiễm nặng, ô nhiễm nhẹ, không ô nhiễm,... ông tìm cách biểu diễn chúng bằng một khái niệm toán học, được gọi là tập mờ. Logic mờ được phát triển từ lý thuyết tập mờ để thực hiện lập luận một cách xấp xỉ thay vì lập luận chính xác. Phương pháp logic mờ nhằm có thể "xử lý" các dữ liệu cao biến, ngôn ngữ, không rõ ràng và không chắc chắn của số liệu hoặc kiến thức và do đó có khả năng đưa ra luồng thông tin lôgic, đáng tin cậy và minh bạch từ số liệu thu thập tới bộ dữ liệu sử dụng trong hệ thống môi trường ứng dụng (Raman \& ctv., 2009).

Đối với bài toán phân lớp dữ liệu như bài toán đánh giá chất lượng nguồn nước, Entropy cực đại là một kỹ thuật rất hữu dụng. Entropy cực đại dùng để ước lượng xác suất các phân phối từ dữ liệu. Tư tưởng chủ đạo của nguyên lý Entropy cực đại là "mô hình phân phối đối với mỗi tập dữ liệu và tập các ràng buộc đi cùng, phải đạt được độ cân bằng/đều nhất có thể". Hay nói cách khác, Entropy là độ đo về tính đồng đều hay tính không chắc chắn của một phân phối xác suất (Alex \& ctv., 2009). Một phân phối xác suất có Entropy càng cao thì phân phối của nó càng đều và khi đó, các yếu tố cao biến có xác suất xuất hiện thấp sẽ không thể ảnh hưởng lớn lên toàn bộ chuỗi dữ liệu (Che, 2013).

Như vậy, chúng tôi đề xuất điều chỉnh công thức xác định chỉ số chất lượng nguồn nước cuối cùng WQI thông qua các xác định các tỷ trọng của các thông số chất lượng trong các nhóm hoá, lý và sinh bằng việc áp dụng phương pháp đánh giá toàn diện mờ.

\section{Vật Liệu và Phương Pháp Nghiên Cứu}

\subsection{Hướng tiếp cận thiết lập công thức đánh giá chất lượng nước mặt khu vực}

Dựa trên công thức tính toán các giá trị chất lượng guồn nước thành phần theo quyết định số 879/QĐ-TCMT cùng với lý thuyết đánh giá toàn diện mờ, các hướng thiết lập công thức đánh giá chất lượng nguồn nước mặt được thực hiện trình tự theo hướng như Hình 1. 


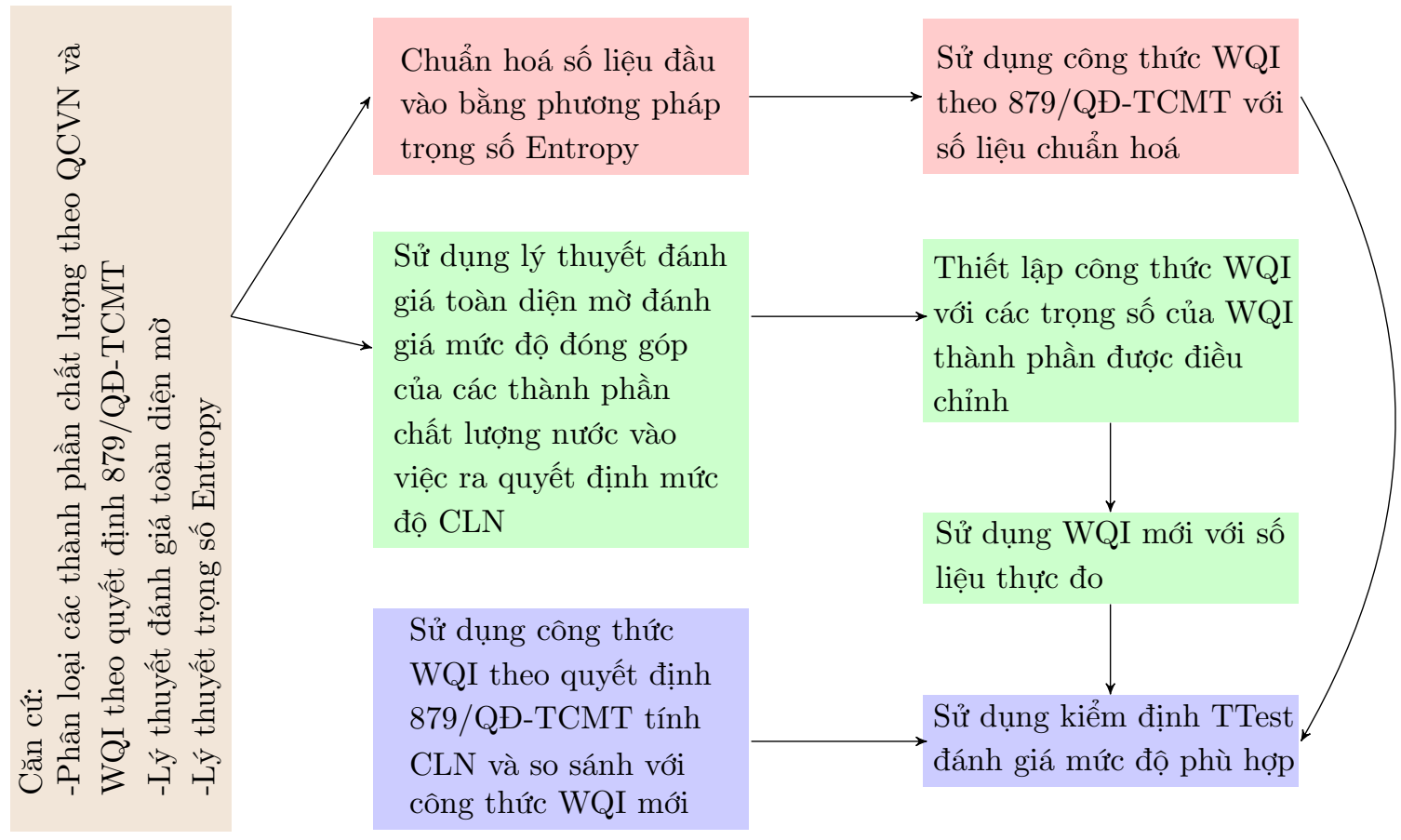

Hình 1. Sơ đồ cách tiếp cận lập công thức đánh giá chất lượng nguồn nước mặt khu vực.

\subsubsection{Cách tiếp cận 1: Ửng dụng phương pháp tính trọng số Entropy "chuẩn hoá" số liệu tính toán chất lượng}

Sử dụng phương pháp tính toán trọng số Entropy loại bỏ (trong trường hợp liệt số liệu lịch sử lớn) hay giảm "mức độ ảnh hưởng" các yếu tố bất thường của số liệu đo đạc. Để sử dụng phương pháp trọng số Entropy, chúng ta sử dụng bộ số liệu đo đạc chất lượng lịch sử. Các bước tính như sau:

Bước 1. Chuẩn hóa dữ liệu gốc. Giả sử ta có $\mathrm{m}$ điểm quan trắc và $\mathrm{n}$ thông số đánh giá lập ma trận dữ liệu gốc $\mathrm{X}$, ma trận chuẩn hoá $\mathrm{R}$ được lập như sau:

$$
\mathrm{r}_{\mathrm{i}, \mathrm{j}}=\frac{\mathrm{x}_{\mathrm{i}, \mathrm{j}}-\operatorname{Min}\left(\sum \mathrm{x}_{\mathrm{i}, \mathrm{j}}\right)}{\operatorname{Max}\left(\Sigma \mathrm{x}_{\mathrm{i}, \mathrm{j}}\right)-\operatorname{Min}\left(\Sigma \mathrm{x}_{\mathrm{i}, \mathrm{j}}\right)}
$$

với thông số tích cực.

$$
\mathrm{r}_{\mathrm{i}, \mathrm{j}}=\frac{\operatorname{Max}\left(\sum \mathrm{x}_{\mathrm{i}, \mathrm{j}}\right)-\mathrm{x}_{\mathrm{i}, \mathrm{j}}}{\operatorname{Max}\left(\sum \mathrm{x}_{\mathrm{i}, \mathrm{j}}\right)-\operatorname{Min}\left(\sum \mathrm{x}_{\mathrm{i}, \mathrm{j}}\right)}
$$

với thông số tiêu cực.

Bước 2. Xác định Entropy theo công thức dưới đây:

$$
\mathrm{H}_{\mathrm{i}}=\frac{1}{\ln (\mathrm{n})} \sum_{\mathrm{j}=1}^{\mathrm{n}} \mathrm{f}_{\mathrm{ij}} \ln (\mathrm{ij})
$$

Trong đó:

$$
f_{i j}=\frac{r_{i j}}{\sum_{j=1}^{n} r_{i j}}, 0 \leq H_{i} \leq 1
$$

Tuy nhiên, khi $f_{i j}=0$ thì $\ln \left(f_{i j}\right)$ không có ý nghĩa. Vì vậy, $\mathrm{f}_{\mathrm{ij}}$ có thể được điều chỉnh như sau:

$$
\mathrm{f}_{\mathrm{ij}}=\frac{1+\mathrm{r}_{\mathrm{ij}}}{\sum_{\mathrm{j}=1}^{\mathrm{n}}\left(1+\mathrm{r}_{\mathrm{ij}}\right)}
$$

Bước 3. Trọng số Entropy được xác định như sau:

$$
\mathrm{w}_{\mathrm{i}}=\frac{1-\mathrm{H}_{\mathrm{i}}}{\mathrm{m}-\sum_{\mathrm{i}=1}^{\mathrm{m}} \mathrm{H}_{\mathrm{i}}}, 0 \leq \mathrm{w}_{\mathrm{i}} \leq 1, \sum_{\mathrm{i}=1}^{\mathrm{m}} \mathrm{w}_{\mathrm{i}}=1
$$

Bước 4. Xác định các giá trị chất lượng sau Entropy. Để xác định các thông số chất lượng được xác định như sau:

$$
\mathrm{x}_{\mathrm{i}, \mathrm{j}}^{\mathrm{E}}=\mathrm{x}_{\mathrm{i}, \mathrm{j}} \times \mathrm{w}_{\mathrm{i}}
$$

Bước 5. Xác định chất lượng nguồn nước mặt theo các bước tính WQI như quyết định 879/QĐTCMT. Kết quả tính toán chất lượng nước theo phương pháp Entropy hoá là WQI ${ }^{\mathrm{E}}$. 
2.1.2. Cách tiếp cận 2: Ứng dụng lý thuyết đánh giá toàn diện mờ với phương pháp tính trọng số Entropy

Để thực hiện việc điều chỉnh công thức đánh giá chất lượng nguồn nước phù hợp với khu vực hay mang tính địa phương, công thức WQI cải tiến dự kiến được thiết lập như sau:

$$
\begin{array}{r}
\mathrm{WQI}^{\mathrm{FCE}}=\mathrm{f}\left(\mathrm{WQI}_{\mathrm{pH}}, \mathrm{WQI}_{\text {hữu cơ }}, \mathrm{WQI}_{\text {lý }},\right. \\
\left.\mathrm{WQI}_{\text {sinh }}\right)
\end{array}
$$

Trong đó:

$$
\begin{array}{r}
\mathrm{WQI}_{\text {hữu cơ }}=\mathrm{f}\left(\mathrm{WQI}_{\% \mathrm{DO}_{\text {bão hoà }}, \mathrm{WQI}_{\mathrm{COD}},},\right. \\
\mathrm{WQI}_{\mathrm{BOD}_{5}}, \mathrm{WQI}_{{\mathrm{N}-\mathrm{NH}_{4}}_{4}}, \\
\left.\mathrm{WQI}_{{\mathrm{P}-\mathrm{PO}_{4}}}\right) \\
\mathrm{WQI}_{\text {lý }}=\mathrm{f}\left(\mathrm{WQI}_{\mathrm{TSS}}, \mathrm{WQI}_{\text {Độ đục }}\right) \\
\mathrm{WQI}_{\text {sinh }}=\mathrm{f}\left(\mathrm{WQI}_{\text {Coliform }}\right)
\end{array}
$$

Các chỉ số chất lượng nước thành phần được tính toán như theo Quyết định số 879/QĐTCMT. Các giá trị chất lượng nguồn nước mặt thuộc các thành phần hữu cơ, vật lý và sinh được tính theo phương pháp đánh giá toàn diện mờ. Các bước tính toán trong phương pháp đánh giá toàn diện mờ như sau:

Bước 1. Chuẩn hoá tập hợp các yếu tố đánh giá U: Trong nghiên cứu, 9 yếu tố chất lượng nước tham gia vào mô hình đánh giá $\mathrm{pH}, \mathrm{DO}, \mathrm{COD}$, $\mathrm{BOD}_{5}, \mathrm{TSS}, \mathrm{N}-\mathrm{NH}_{4}, \mathrm{P}_{-} \mathrm{PO}_{4}$, Tổng Coliform và độ đục. Tập hợp các yếu tố đánh giá U có thể viết như sau (Panchal, 2011; Che, 2013):

$$
\begin{aligned}
& \mathrm{U}_{\mathrm{mn}}=\left\{\mathrm{pH}, \mathrm{DO}, \mathrm{COD}, \mathrm{BOD}_{5}, \mathrm{TSS},\right. \\
& \mathrm{N}-\mathrm{NH}_{4}, \mathrm{P}_{-} \mathrm{PO}_{4}, \text { Coliform, } \\
& \text { Độ đục\} }
\end{aligned}
$$

Với: m là số mẫu/số điểm quan trắc, $n$ là thông số.

Bước 2. Xây dựng hệ thống phân bậc cho các yếu tố đánh giá: Hệ thống phân bậc đánh giá chất lượng nước trong nghiên cứu này chia làm 5 bậc, dựa trên các bảng tính toán chỉ số phụ CLN theo các thông số đánh giá trong hướng dẫn của Quyết định 879/QĐ-TCMT. Do vậy, tập hợp hệ thống phân bậc cho các yếu tố tham gia mô hình đánh giá là $\mathrm{V}_{\mathrm{kn}}=\mathrm{I}, \mathrm{II}, \mathrm{III}, \mathrm{IV}, \mathrm{V} ; \mathrm{k}=5$ bậc và $\mathrm{n}=9$ thông số. Năm bậc chất lượng nước (hay ô nhiễm) theo ngôn ngữ tự nhiên là: I - Chưa ô nhiễm, II - Ô nhiễm nhẹ, III - Ô nhiễm trung bình, IV Ô nhiễm nặng, V - Ô nhiễm nghiêm trọng. Các giá trị phân chia 5 bậc ô nhiễm cho các thông số tham gia đánh giá trình bày ở Bảng 2 .

Bước 3. Xác định trọng số của các thông số thành phần (Ji-hong \& ctv., 2009; Jun-Jian \& ctv., 2008): Trọng số được xác định theo phương pháp Entropy. Phương pháp này được ứng dụng để đo lường kích thước của thông tin, càng nhiều thông tin chứa đựng trong một chỉ thị đặc trưng thì ảnh hưởng của chỉ thị đó trong việc ra quyết định càng trở nên quan trọng. Do đó, Entropy cũng được áp dụng để gán trọng số cho các chỉ thị môi trường.

Bước 4. Xác định các thông số chất lượng nguồn nước phục vụ cho đánh giá mức độ chất lượng. Sau khi xác định được giá trị trọng số Entropy, các giá trị của các thông số chất lượng nguồn nước (được đo đạc) sẽ được làm "mờ" các tính chất bất lợi cho đánh giá như tính cao biến, không rõ ràng... Dựa vào các giá trị "mới" của các thông số chất lượng chúng ta có thể xác định được mức độ/bậc chất lượng môi trường nước của khu vực xem xét về phương diện không gian và thời gian (quý, năm, nhiều năm...) tùy thuộc nhu cầu của các nhà quản lý môi trường khi đưa ra quyết định (Cách tính tương tự như hướng tiếp cận 1).

Bước 5: Dựa trên bảng phân loại chất lượng nguồn nước, bằng phương pháp xác suất thông kê xác định mức độ "quan trọng" của các thông số chất lượng, nhóm chất lượng (hoá, sinh, lý) thông qua mức độ "đóng góp" của chúng cho từng bậc chất lượng. Dựa trên tập hợp mức độ đóng góp của các nhóm chất lượng này (hoá, sinh, lý) có mức độ lệch chuẩn nhỏ nhất (thường xét trường hợp cận nhỏ nhất) từ đó xác định các hệ số cho công thức $9-12$.

Như vậy công thức 9 - 12 (Đánh giá chất lượng nguồn nước theo chỉ số $\mathrm{WQI}^{\mathrm{FCE}}$ cải tiến) được xác định như sau:

$$
\begin{array}{r}
\mathrm{WQI}_{\mathrm{FCE}}=\frac{\mathrm{WQI}_{\mathrm{pH}}}{100} \times\left(\mathrm{WQI}_{\mathrm{a}}^{\mathrm{ra}}\right. \\
\times \mathrm{WQI}_{\mathrm{b}}^{\mathrm{rb}} \\
\left.\times \mathrm{WQI}_{\mathrm{c}}^{\mathrm{rc}}\right)
\end{array}
$$

Trong đó:

$$
\begin{array}{r}
\mathrm{WQI}_{\mathrm{a}}=\mathrm{a}_{1} \mathrm{WQI}_{\mathrm{DO}}+\mathrm{a}_{2} \mathrm{WQI}_{\mathrm{BOD}_{5}}+\mathrm{a}_{3} \mathrm{WQI}_{\mathrm{COD}} \\
+\mathrm{a}_{4} \mathrm{WQI}_{\mathrm{N}-\mathrm{NH}_{4}}+\mathrm{a}_{5} \mathrm{WQI}_{{\mathrm{P}-\mathrm{PO}_{4}}}
\end{array}
$$




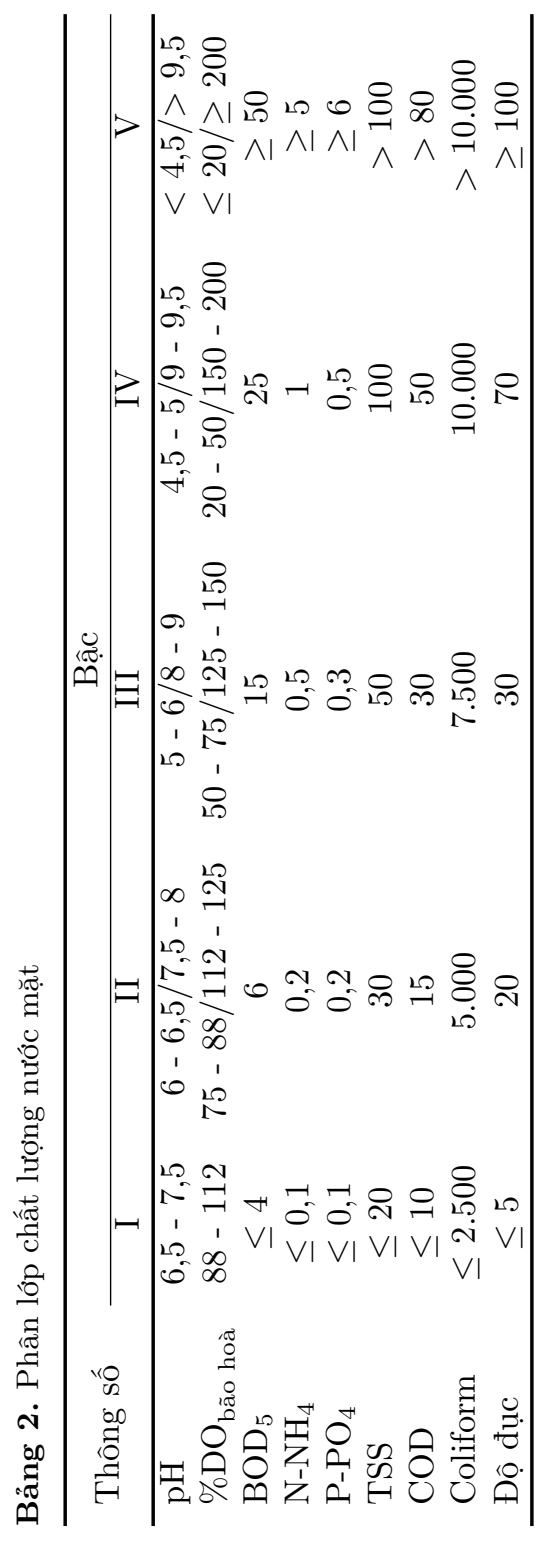

$$
\begin{aligned}
& \mathrm{WQI}_{\mathrm{b}}=\mathrm{b}_{1} \mathrm{TSS}_{\mathrm{DO}}+\mathrm{b}_{2} \mathrm{WQI}_{\text {Độ đục }} \\
& \mathrm{WQI}_{\mathrm{c}}=\mathrm{WQI}_{\text {Coliform }} \\
& \mathrm{r}_{\mathrm{a}}, \mathrm{r}_{\mathrm{b}}, \mathrm{r}_{\mathrm{c}} \text { là các tỷ trọng với } \mathrm{r}_{\mathrm{a}}+\mathrm{r}_{\mathrm{b}}+\mathrm{r}_{\mathrm{c}}=1 \\
& \mathrm{a}_{1}, \ldots, \mathrm{a}_{5} ; \mathrm{b}_{1}, \mathrm{~b}_{2} \text { là hệ số của các thông số với: } \\
& \mathrm{a}_{1}+\mathrm{a}_{2}+\mathrm{a}_{3}+\mathrm{a}_{4}+\mathrm{a}_{5}=1 ; \mathrm{b}_{1}+\mathrm{b}_{2}=1
\end{aligned}
$$

Các hệ số trên phản ánh tính khu vực. Dựa vào liệt số liệu các thông số và chất xác định chất lượng nguồn nước lịch sử, chúng ta xác định các hệ số trên. Để là rõ hơn, chúng ta sử dụng liệt số liệu đo đạc về chất lượng nguồn nước mặt trên địa bàn tỉnh Vĩnh Long để xác lập công thức đánh giá chất lượng nước khu vực. Kết quả được thể hiện ở dưới đây.

\section{Kết Quả và Thảo Luận}

Thành phố Vĩnh Long là một trong những địa phương nằm trong khu vực có mật độ sông kênh rạch rất dày đặc, chằng chịt và liên thông với nhau. Tuy nhiên, vấn nạn ô nhiễm môi trường nước mặt khu vực do rác thải, nước thải từ hoạt động sản xuất và sinh hoạt dọc các sông, kênh, rạch có chiều hướng ngày một trầm trọng hơn. Theo kết quả quan trắc chất lượng nước sông được Trung tâm quan trắc môi trường Thành phố Vĩnh Long thực hiện hàng năm tại các vị trí tập trung dân cư và khu công nghiệp, thì chất lượng nước các con sông chính đã và đang bị ô nhiễm từ vừa đến trầm trọng.

Hiện tại Sở Tài nguyên Môi trường tỉnh Vĩnh Long thường xuyên công bố chất lượng nước mặt, nước ngầm trên địa bàn Tỉnh, trong đó có khu vực Thành phố Vĩnh Long, tại các vị trí dọc các sông Tiền, sông Cái Cam, sông Cái Côn, sông Cầu Lầu, sông Cầu Vồng và sông Long Hồ. Các vị trí này được thể hiện trong Hình 2. Số liệu sử dụng tính toán là số liệu quan trắc chất lượng nguồn nước trên các sông chính khu vực Thành phố Vĩnh Long từ 2012 đến 2017. Ví dụ số liệu chất lượng nước mặt năm 2016 được trình bày trong Bảng 3.

\subsection{Các bước thiết lập công thức đánh giá CLN khu vực Thành phố Vĩnh Long}

Các bước 1, 2 được thực hiện tại các Bảng 2 và 3 .

Bước 3: Thiết lập ma trận chuẩn hoá số liệu gốc (bước 1 trong cách tiếp cận 1). Bảng ma trận chuẩn hoá cùng giá trị trọng số Entropy (tương đương bước 2, 3 trong tiếp cận 1) như Bảng 5 


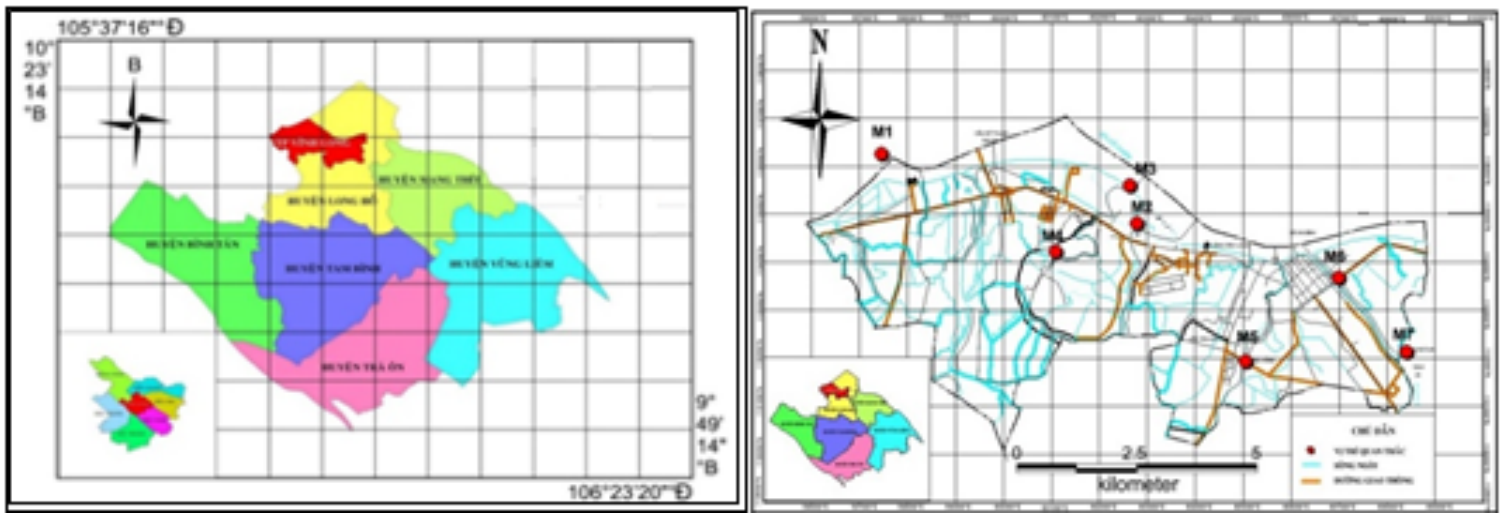

Hình 2. Bản đồ hành chính Thành phố Vĩnh Long và vị trí quan trắc (Sở Tài nguyên Môi trường tỉnh Vĩnh Long, 2016).

cùng với Bảng 4 thống kê các giá trị trung bình, $\max , \min$.

Bước 4: Với kết quả tính toán trọng số Entropy, chúng ta tính toán giá trị các thông số chất lượng cho đánh giá bậc chất lượng (tương dương bước 4 trong cách tiếp cận 1). Bảng thông số chất lượng (Bảng 6) và bảng đánh giá bậc chất lượng (Bảng 7).

Bước 5: Thiết lập công thức đánh giá chất lượng nguồn nước khu vực. Từ công thức 13 , chúng ta xác định các hệ số thông qua việc xác định mức độ đóng góp của các thông số chất lượng và các nhóm thông số trong từng mức độ chất lượng nguồn nước. Các bước tính toán được thống kê trong các Bảng 8, 9 và 10 .

Công thức đánh giá chất lượng nước khu vực cho tỉnh Vĩnh Long:

$$
\begin{aligned}
& \mathrm{WQI}_{\mathrm{E}}=\frac{\mathrm{WQI}_{\mathrm{pH}}}{100} \times\left[\left(0.26 \mathrm{WQI}_{\mathrm{DO}}\right.\right. \\
& +0.16 \mathrm{WQI}_{\mathrm{COD}}+0.14 \mathrm{WQI}_{\mathrm{BOD}} \\
& \left.+0.37 \mathrm{WQI}_{\mathrm{N}-}+0.07 \mathrm{WQI}_{\mathrm{P}-}\right)^{0.53} \\
& \times\left(0.53 \mathrm{WQI}_{\mathrm{TSS}}+0.47 \mathrm{WQI}_{\text {Độ đục }}\right)^{0.35} \\
& \left.\times \mathrm{WQI}_{\text {Coliform }}^{0.12}\right]
\end{aligned}
$$

Như vậy, công thức đánh giá chất lượng nguồn nước khu vực có nhiều khác biệt so với công thức WQI theo quyết định 879/QĐ-TCMT như: (i) các giá trị mũ của các nhóm khác $(1 / 3)$, (ii) các hệ số của các thông số chất lượng trong các nhóm cũng khác nhau. Các yếu tố này thể hiện đặc điểm đặc trưng về thành phần chất lượng của khu vực (thông qua phân tích chuỗi số liệu đo đạc lịch sử) như yếu tố hữu cơ có tính quyết định chất lượng là
N-NH 4 , yếu tố TSS quan trọng nhất trong nhóm vật lý. Nhóm sinh (coliform) là thứ yếu khi mức độ luân chuyển nước mặt trong hệ thống sông kênh khu vực tốt cũng như yếu tố hệ sinh thái khu vực ổn định dẫn đến mức độ coliform không có những đột biến hay quá lớn để "dẫn dắt" bậc của chất lượng nguồn nước khu vực.

So sánh giá trị chất lượng nguồn nước cũng như bậc mức độ tại khu vực tỉnh Vĩnh Long (Bảng 11), bậc mức độ chất lượng giữa 2 cách tiếp cận và phương pháp tính $\mathrm{WQI}^{879}$ theo quyết định 879/QĐ-TCMT không khác biệt nhau, nhưng giá trị chất lượng nguồn nước tính theo phương pháp tiếp cận 2 (WQI $\left.{ }^{\mathrm{FEC}}\right)$ đã thể hiện mức độ cải thiện tốt hơn.

\subsection{Kiểm định thống kê $T$ - test}

Kiểm định thống kê T- test từng cặp giá trị WQI theo quyết định số 879/QĐ-TCMT với thông số đo đạc thực tế (WQI ${ }^{879}$ ), các thông số đã được entropy hoá (WQI ${ }^{\text {Entropy }}$ ) và công thức điều chỉnh theo thuật toán đánh giá toàn diện mờ (WQI ${ }^{\mathrm{FCE}}$ ) được tổng hợp theo Bảng 12.

Với bảng kiểm định T-test, việc so sánh các chuỗi kết quả CLN WQIFCE và WQI879 khi giá trị $\mathrm{P}(\mathrm{T} \leq \mathrm{t})$ one-tail lớn $0,05(5 \%)$ điều này nói lên có sự khác biệt về định lượng giữa chúng. Trường hợp so sánh chuỗi CLN các năm giưa $\mathrm{WQI}^{879}$ và WQI ${ }^{\text {Entropy }}$ không có sự khác biệt. Cách tính CLN theo $\mathrm{WQI}^{879}$ và $\mathrm{WQI}^{\mathrm{FCE}}$ có cùng xu hướng khi (|t Stat $|<| \mathrm{t}$ Critical $\mid)$.

Với cách tính CLN theo công thức điều chỉnh WQI ${ }^{\mathrm{FEC}}$ so với WQI ${ }^{879}$ đã có sự khác biệt trung bình hay có sự khác biệt về định lượng. Điều này 

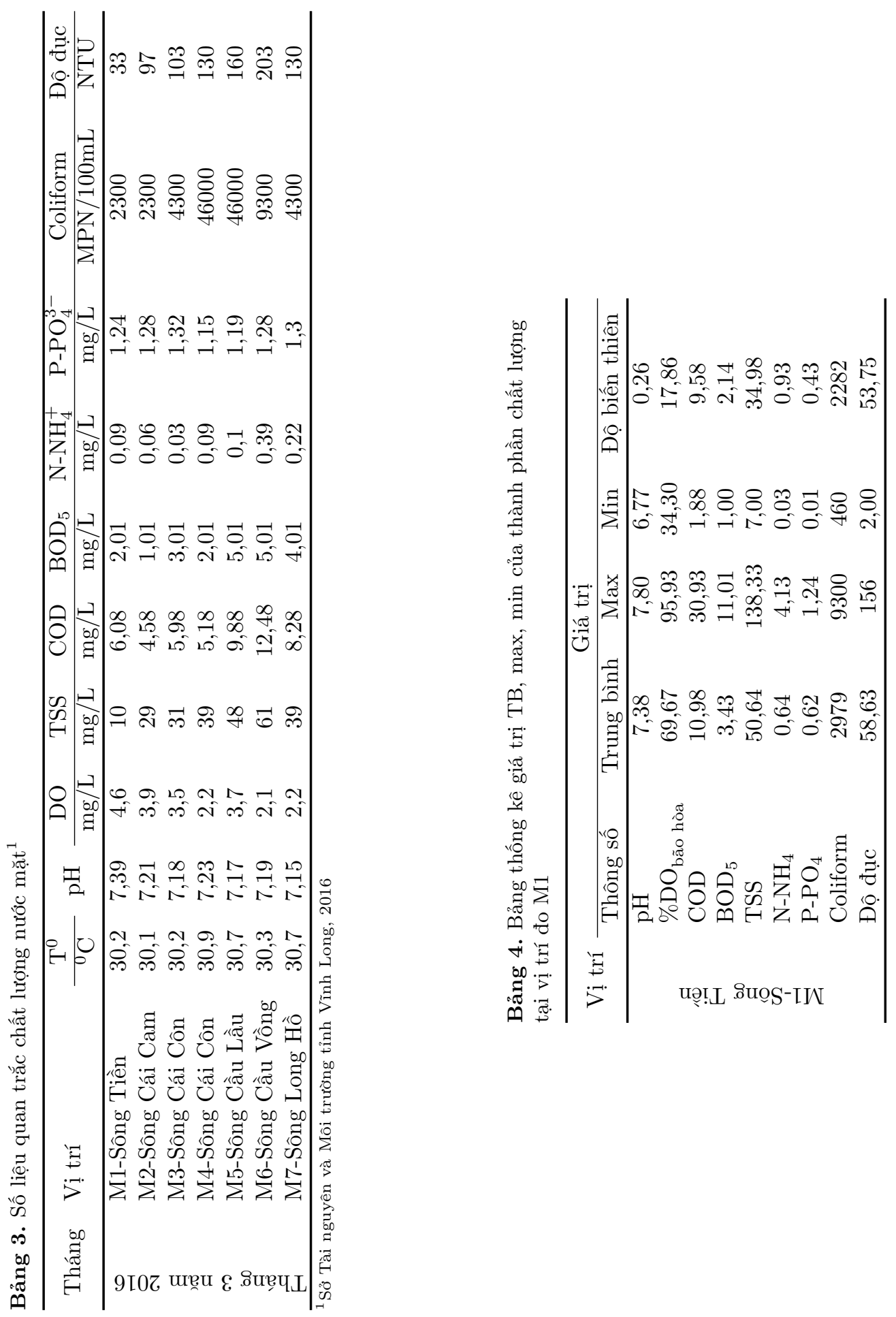

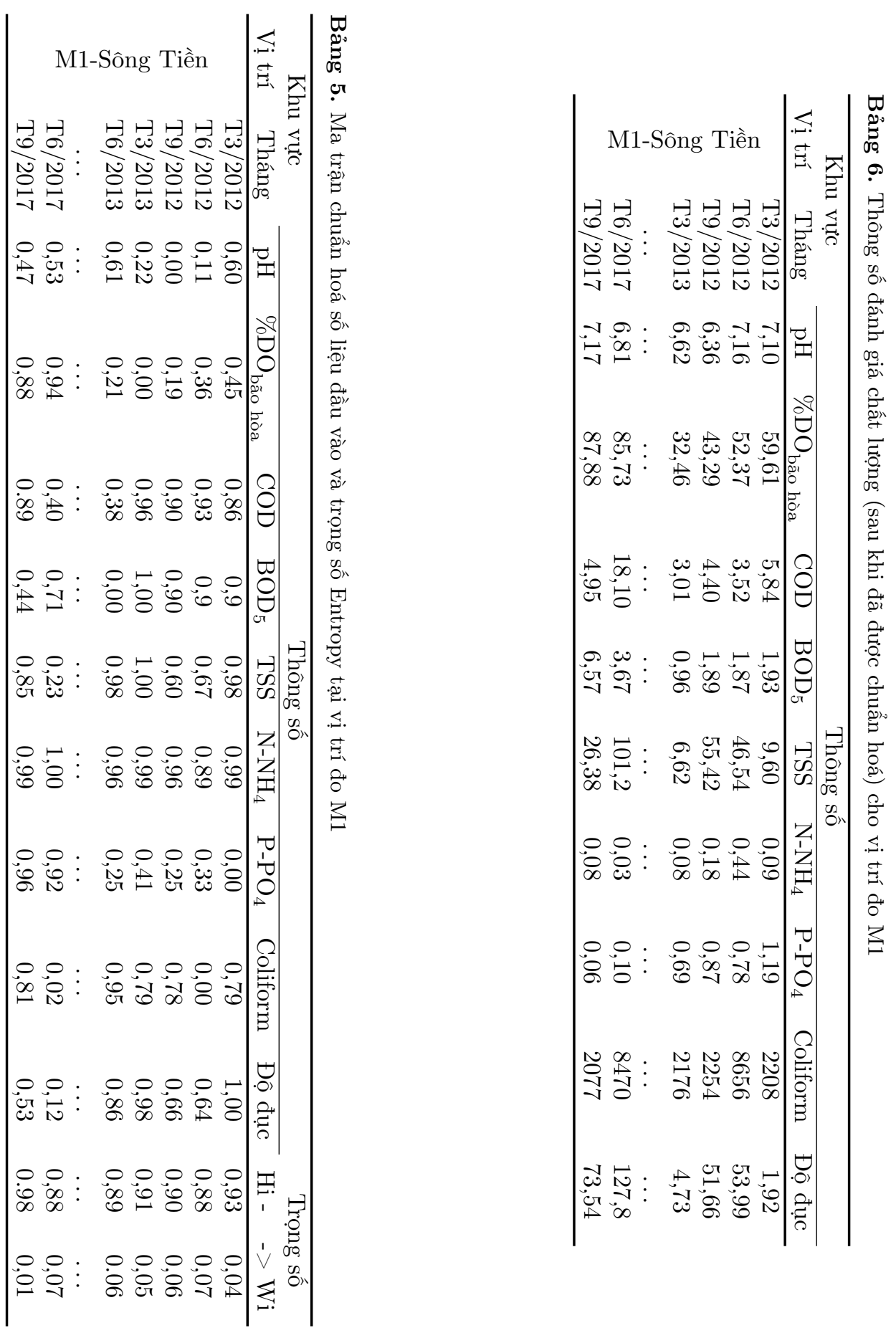

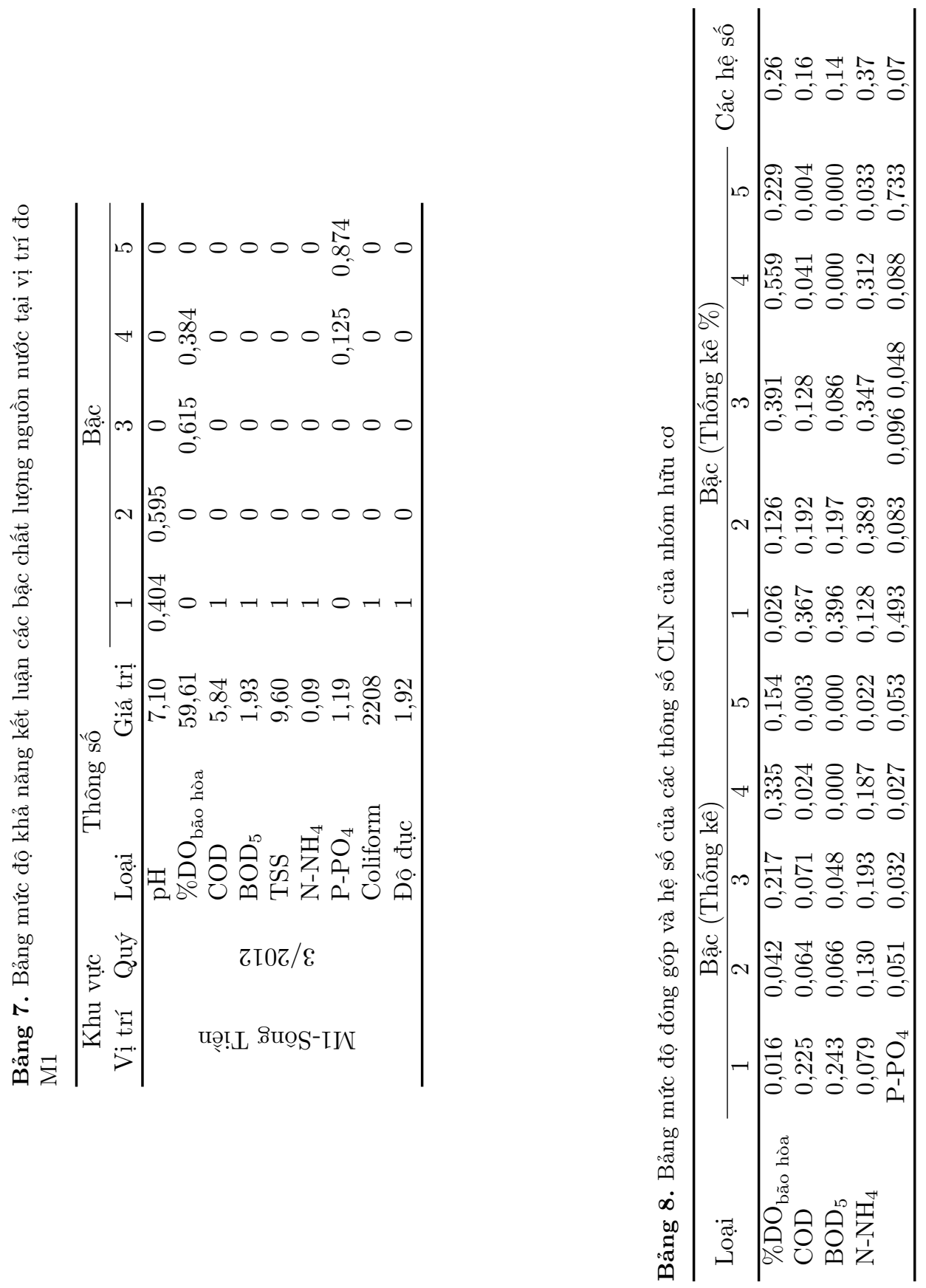
Bảng 9. Bảng mức độ đóng góp và hệ số của các thông số CLN của nhóm Vật lý

\begin{tabular}{|c|c|c|c|c|c|c|c|c|c|c|c|}
\hline \multirow{2}{*}{ Loại } & \multicolumn{5}{|c|}{ Bậc (Thống kê) } & \multicolumn{5}{|c|}{ Bậc (Thống kê \%) } & \multirow{2}{*}{$\begin{array}{l}\text { Các } \\
\text { hệ số }\end{array}$} \\
\hline & 1 & 2 & 3 & 4 & 5 & 1 & 2 & 3 & 4 & 5 & \\
\hline$\overline{\mathrm{TSS}}$ & 0,086 & 0,115 & 0,193 & 0,155 & 0,048 & 0,554 & 0,467 & 0,594 & 0,469 & 0,293 & 0,53 \\
\hline Độ đục & 0,069 & 0,131 & 0,132 & 0,176 & 0,115 & 0,446 & 0,533 & 0,406 & 0,531 & 0,707 & 0,47 \\
\hline
\end{tabular}

Bảng 10. Bảng mức độ đóng góp và hệ số của các nhóm thông số CLN

\begin{tabular}{|c|c|c|c|c|c|c|c|c|c|c|c|}
\hline \multirow{2}{*}{ Nhóm } & \multicolumn{5}{|c|}{ Bậc (Thống kê) } & \multicolumn{5}{|c|}{ Bậc (Thống kê \%) } & \multirow{2}{*}{ Số mũ } \\
\hline & 1 & 2 & 3 & 4 & 5 & 1 & 2 & 3 & 4 & 5 & \\
\hline Hóa & 0,689 & 0,483 & 0,583 & 0,606 & 0,673 & 0,689 & 0,483 & 0,583 & 0,606 & 0,673 & 0,53 \\
\hline Lý & 0,175 & 0,354 & 0,342 & 0,335 & 0,163 & 0,175 & 0,354 & 0,342 & 0,335 & 0,163 & 0,35 \\
\hline Sinh & 0,137 & 0,163 & 0,075 & 0,059 & 0,164 & 0,137 & 0,163 & 0,075 & 0,059 & 0,164 & 0,12 \\
\hline
\end{tabular}

Bảng 11. Bảng phân chất lượng nguồn nước khu vực theo các cách tính khác nhau

\begin{tabular}{|c|c|c|c|c|c|}
\hline \multicolumn{3}{|c|}{ Nam trung bình } & \multicolumn{3}{|c|}{ M2-Sông Cái Cam } \\
\hline Tháng & $\mathrm{WQI}^{879}$ & $\mathrm{WQI}^{\mathrm{FCE}}$ & Tháng & $\mathrm{WQI}^{879}$ & $\mathrm{WQI}^{\mathrm{FCE}}$ \\
\hline T3/2012 & 69 & 74 & T3/2012 & 87 & 86 \\
\hline T6/2012 & 39 & 49 & T6/2012 & 66 & 63 \\
\hline T9/2012 & 54 & 59 & T9/2012 & 68 & 63 \\
\hline T3/2013 & 78 & 79 & T3/2013 & 86 & 82 \\
\hline T6/2013 & 67 & 68 & T6/2013 & 72 & 67 \\
\hline Т9/2013 & 37 & 36 & Т9/2013 & 18 & 15 \\
\hline $\mathrm{T} 3 / 2014$ & 76 & 80 & $\mathrm{~T} 3 / 2014$ & 92 & 91 \\
\hline T6/2014 & 68 & 72 & T6/2014 & 56 & 72 \\
\hline T9/2014 & 52 & 54 & Т9/2014 & 66 & 60 \\
\hline T3/2015 & 83 & 77 & T3/2015 & 91 & 82 \\
\hline T6/2015 & 69 & 69 & T6/2015 & 71 & 71 \\
\hline T9/2015 & 54 & 56 & Т9/2015 & 52 & 58 \\
\hline T3/2016 & 64 & 68 & T3/2016 & 71 & 75 \\
\hline $\mathrm{T} 6 / 2016$ & 69 & 68 & $\mathrm{~T} 6 / 2016$ & 65 & 68 \\
\hline Т9/2016 & 51 & 53 & T9/2016 & 55 & 59 \\
\hline T3/2017 & 68 & 65 & T3/2017 & 67 & 63 \\
\hline T6/2017 & 46 & 54 & T6/2017 & 53 & 59 \\
\hline T9/2017 & 56 & 66 & Т9/2017 & 18 & 47 \\
\hline
\end{tabular}

WQI ${ }^{879}-$ CLN tính theo công thức trong 879/QĐ-TCMT với số liệu thực đo; WQI ${ }^{\mathrm{FCE}}-\mathrm{CLN}$ tính theo công thức số 14 với số liệu thực đo; Xanh da trời - Chưa ô nhiễm, xanh lá cây - Ô nhiễm nhẹ, Vàng - Ô nhiễm trung bình, cam - Ô nhiễm nặng, Đỏ - Ô nhiễm nghiêm trọng.

Bảng 12. Kiểm định T-test cho cặp chuỗi kết quả CLN các tháng

\begin{tabular}{lccc}
\hline & WQI $^{879}$ & WQI $^{\text {Entropy }}$ & WQI $^{\mathrm{FCE}}$ \\
\hline Trung bình & 62,6 & 63,8 & 63,91 \\
Phương sai & 144,6 & 129,4 & 87,28 \\
Số quan sát & 22 & 22 & 22 \\
Tương quan Pearson & & 1,00 & 0,879 \\
Giả thuyết sự khác biệt trung bình $(0 / 1)$ & 0 & 0 \\
df & & 21 & 21 \\
t Stat & & $-6,046$ & $-1,014$ \\
P $(T \leq t)$ one-tail & & 0,000 & 0,161 \\
t Critical one-tail & 1,323 & 1,721 \\
P $(T \leq t)$ two-tail & 0,000 & 0,322 \\
t Critical two-tail & 1,721 & 2,080 \\
\hline
\end{tabular}


nói lên, các giá trị CLN theo công thức điều chỉnh đã được "điều chỉnh" theo hướng phù hợp với thực tế sử dụng nước của khu vực (các giá trị CLN theo tháng và năm đều ở mức chất lượng tốt hơn).

\subsection{Hiện trạng chất lượng nguồn nước khu vực thành phố Vĩnh Long}

Căn cứ vào công thức điều chỉnh WQI số 17 , CLN mặt hệ thống sông kênh trên địa bản Thành phố Vĩnh Long năm 2017 được đánh giá như sau. Kết quả đánh giá được thể hiện Hình 3 (điển hình sông Tiền và sông Cầu Vồng - nội tỉnh).
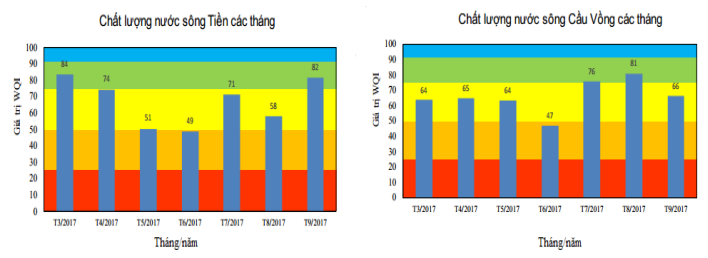

Hình 3. Chất lượng nước mặt trung bình tháng trong năm 2017. Xanh da trời - Chưa ô nhiễm, xanh lá cây - Ô nhiễm nhẹ, vàng - Ô nhiễm trung bình, cam - Ô nhiễm nặng, đỏ - Ô nhiễm nghiêm trọng.

Như vậy, CLN mặt sông Tiền - đoạn chảy qua thành phố Vĩnh Long vẫn đảm bảo cho tưới tiêu, giao thông thuỷ và cấp nước sinh hoạt (có xử lý trước khi cấp) do mức chất lượng là ô nhiễm nhẹ đến vừa tại hầu hết các điểm đo. CLN sông Cầu Vồng đang được cải thiện rõ rệt với mức ô nhiễm từ nhẹ đến vừa. Điều này hợp lý so với thực tế sử dụng nước khu vực này, khi diện tích sản xuất nông nghiệp đang giảm, nhiều khu công nghiệp xuất hiện và tốc độ đô thị hoá nhanh, các kênh này dần trở thành nơi chứa và tiêu thoát nước thải.

\section{Kết Luận}

Qua ví dụ tính toán chất lượng nguồn nước các sông trên địa bàn Thành phố Vĩnh Long các năm 2012 - 2017 theo chỉ số đánh giá WQI, cho thấy chất lượng nguồn nước đang bị ô nhiễm với các mức nhẹ đến vừa và biến động chất lượng trong năm. Đánh giá chất lượng nguồn nước theo công thức chỉ số WQI điều chỉnh theo phương pháp đánh giá toàn diện mờ (FCE) có cơ sở lý luận logic, rõ ràng và chính xác hơn (khi giảm thiểu ảnh hưởng/định hướng của các thông số cao biến như coliform) so với công thức tính của quyết định 879/QĐ-TCMT. Kết quả đánh giá chất lượng nguồn nước mặt theo công thức điều chỉnh bước đầu cho thấy sự phù hợp với hiện trạng sử dụng nước năm 2017 của địa phương và đúng với định hướng điều chỉnh phương pháp tính WQI của Tổng cục môi trường (với quyết dịnh 711/QĐ-TCMT).

Tuy nhiên, để khẳng định cũng như áp dụng rộng rãi chỉ số này cần phải tiến hành nghiên cứu khoa học cụ thể và áp dụng cho nhiều khu vực khác nhau nhằm hoàn thiện phương pháp tính toán.

\section{Tài Liệu Tham Khảo (References)}

Alex. W. D., van Gelder,. P. H. A. J. M., \& Vrijling, J. K. (2009). Risk assessment of petroleum pipelines using a combined analytical hierarchy process - Fault tree analysis (AHP-FTA). Cham, Switzerland: Springer International Publishing.

Che, L. D. (2013). Application of "fuzzy" comprehensive evaluation method in the model of water quality assessment of Saigon River flowing through Binh Duong Province. Journal of Environment 6, 30-42.

Bui, H. V., \& Tran, N. M. U. (2017). Evaluation of the current status of surface water quality at main canal in Vinh Long province (Unpublished master's thesis). University of Science, Ho Chi Minh City, Vietnam.

Ji-hong, Z., Cai-lian, H., Jun-guang, Z., \& Li, P. (2009). Water quality assessment of Zhanghe River based on fuzzy evaluation method. Retrieved December 20, 2009, from https://ieeexplore.ieee.org/document/5406823.

Jun-Jian, Q., Xin-Wu, Z., \& Yan-Rui, Z. (2008). The application offuzzy comprehensive evaluationn on the water quality of Changiiang River 2008. Retrieved August 12, 2008, from http://xplorebcpaz.ieee.org/stamp/stamp.jsp?tp=\&ar number-4620637.

Panchal, J. (2011). Fuzzy classificationan overview. Cham, Switzerland: Springer International Publishing.

Raman, B. V., Reinier, B., \& Mohan, S. (2009). Fuzzy Logic Water Quality Index and Importance of Water Quality Parameters. Air, Soil and Water Research 2, 51-59.

VEA (Vietnam Environment Administration). (2011). Decision No. 879/QD-TCMT in according to WQI manual calculation handbook. Ha Noi, Vietnam: Ministry of Natural Resource and Environment. 